\title{
Incubation Dose for Ion Beam Induced Anisotropic Growth of Amorphous Alloys: Insight into Amorphous State Modifications
}

\author{
A. I. Ryazanov, ${ }^{1}$ H. Trinkaus, ${ }^{2}$ and A.E. Volkov ${ }^{1, *}$ \\ ${ }^{1}$ Russian Research Center "Kurchatov Institute," 123182 Moscow, Russia \\ ${ }^{2}$ Institut für Festkörperforschung, Forschungszentrum Jülich, D-52425 Jülich, Germany
}

(Received 17 September 1999)

\begin{abstract}
Under energetic ion bombardment, amorphous materials deform plastically in the form of anisotropic growth. At medium electronic stopping power ( 5 to $30 \mathrm{keV} / \mathrm{nm}$ ) this phenomenon starts only after a certain incubation dose depending on values of the electronic stopping power and temperature. This delay is modeled on the basis of the assumption of a drastic irradiation induced viscosity reduction, resulting from accumulation of atomic displacements in the matrix and local material heating in the heavy ion track. A simple analytical expression is derived which is in a good agreement with experimental data for the amorphous alloy $\mathrm{Fe}_{85} \mathrm{~B}_{15}$.
\end{abstract}

PACS numbers: $61.80 . \mathrm{Jh}$

In the amorphous (or glassy) state, solid materials are far from equilibrium. An important aspect of this nonequilibrium solid state is that it may be realized, at least within short time periods, in a broad spectrum of metastable states with different degrees of relative stability, relaxation times, and activation barriers for these relaxations [1].

There are several techniques to amorphize crystalline and to treat amorphous materials, but bombardment with heavy ions is certainly the method where the most extreme conditions can be realized due to the deposited energy being extremely localized and having a high density. Thus, displacement cascades and electronic excitations along the ion trajectory induce rapid heating and cooling in sub-ns time scales and at rates of up to $10^{15} \mathrm{~K} / \mathrm{s}$. Under such conditions, an amorphous material may be investigated at temperatures where it would recrystallize within the time scales of "classical" methods. Bombardment of amorphous materials with heavy ions can also considerably influence the relaxation kinetics of the metastable states and may be considered to provide a deeper understanding of the dynamics of the amorphous state.

Energetic heavy ions penetrating a solid lose their energy by quasielastic collisions with the target atoms and by electronic excitations characterized, respectively, by "nuclear" and "electronic" stopping powers, $S_{n}$ and $S_{e}$. We are interested here in ion energies above $1 \mathrm{MeV} / \mathrm{amu}$ where $S_{e}$ dominates the stopping power. In this energy range, heavy ions with sufficiently high electronic stopping power $\left(S_{e}>5 \mathrm{keV} / \mathrm{nm}\right)$ induce in amorphous materials at low temperatures $\left(T_{\text {irr }} \leq 200-300 \mathrm{~K}\right)$ two striking deformation phenomena: (i) anisotropic expansion ("growth") of unstressed amorphous foil samples perpendicular to the ion-beam direction [2-8] and (ii) creep of stressed amorphous samples with creep or stress relaxation rates [7-13] which are larger by orders of magnitude than those in crystalline or polycrystalline materials.

At medium electronic stopping power $(5-30 \mathrm{keV} / \mathrm{nm})$ the occurrence of both deformation phenomena, i.e., (i) and (ii), requires some incubation fluence (dose) $\phi_{c}$ which depends on the electronic and nuclear stopping powers, irradiation temperature, and amorphous material properties $[2,3,5,6]$.

Recently, ion-beam induced anisotropic growth [14-17] and creep (or stress relaxation) [16-18] of amorphous materials have been described in terms of viscous flow and shear stress relaxation in locally heated and damaged regions in the vicinity of the ion trajectories. In the case of anisotropic growth, the shear stress, assumed to relax upon temporal local heating in the vicinity of the projectile trajectory, is due to the thermal expansion in the heated cylindrical track whereas in the creep (stress relaxation) it is given simply by the externally applied macroscopic stress. In these models, the local heating is assumed to result from collision cascades as well as electronic excitations. In recent years the latter possibility has been discussed intensively [19-24].

The problem of the incubation fluence was not investigated in detail in Refs. [14,15]. In Ref. [15] it was only predicted that no incubation dose would be necessary if the local heating in the track, i.e., the energy deposition, was sufficiently high as expected for very high electronic stopping power. This prediction has been confirmed experimentally [7,8]. In Ref. [14] a significant decrease of the material viscosity in addition to the effect of local heating was assumed to be necessary for the onset of substantial anisotropic growth. But the mechanism of this decrease, which we consider to be responsible for the incubation dose, was not investigated there in detail. In this paper we make an attempt to model the dependence of the incubation fluence on the electronic stopping power $\left(S_{e}\right)$, the nuclear stopping power $\left(S_{n}\right)$, and the irradiation temperature ( $T_{\mathrm{irr}}$ ) for medium values of $S_{e}$ between 5 and $30 \mathrm{keV} / \mathrm{nm}$ where the transition from a low dose incubation stage with negligible growth to unsaturated linear growth is relatively sharp.

It is useful here to recall the scenario of excitation and relaxation processes induced by an energetic heavy ion penetrating a solid. Electronic excitations along the ion 
track occur in less than $10^{-16} \mathrm{~s}$ and the electronic energy deposited per unit length is given by the electronic stopping power $S_{e}$. Thermalization in the excited electronic subsystem takes place within about $10^{-15} \mathrm{~s}$ and the maximum electronic temperature which results may reach values substantially above the Fermi temperature while the temperature in the subsystem of the atomic cores is still close to that of the surroundings. Because of this temperature difference, energy, while spreading diffusionally in the electronic system, is transferred from there to the atomic system within characteristic times, $\tau_{e a}\left(S_{e}\right)$, between $10^{-14}$ and $10^{-11} \mathrm{~s}$ depending on the magnitude and mode of the electron-ion coupling [20-25]. The maximum temperature increase in the atomic system, $\Delta T_{0}$, attained by this heating process depends on the deposited energy, $S_{e}$, and on the width of its distribution, $R_{T}\left(\tau_{e a}\right) \approx 4 D_{e} \tau_{e a}$ (where $D_{e}$ is the electronic thermal diffusivity), reached at a time of the order of $\tau_{e a}$. In the high temperature limit of the specific heat of the atomic system, $C=3 n_{a} k_{B}$, where $n_{a}$ is the atomic density and $k_{B}$ is the Boltzmann constant, this relation can be written as

$$
\Delta T_{0}=S_{e} /\left(3 \pi n_{a} k_{B} R_{T}^{2}\right) .
$$

Taking into account values obtained (see below) of the heating radius $R_{T} \approx 20-30 \mathrm{~nm}$, one concludes that the initial material heating in the track ranges from 100 to $1000 \mathrm{~K}$ depending on the electronic energy loss of the penetrating ion. After the maximum temperature is passed the heated atomic system cools down by heat diffusion within a time scale given by $\tau_{\text {th }} \approx R_{T}^{2} / D_{a}$ which ranges from a few $10^{-11} \mathrm{~s}$ to $10^{-9} \mathrm{~s}$ depending on the $R_{T}$ and the atomic thermal diffusivity $D_{a}$ of the amorphous material.

Substantial shear stress relaxation and the associated plastic deformation in the heated region would take place if the characteristic time for shear stress relaxation, $\tau_{\sigma}=$ $\eta / \mu$ ( $\eta$ : viscosity, $\mu$ : shear modulus) was of the order or shorter than the local heating "lifetime" $\tau_{\text {th }}$, which may be expressed for the given $S_{e}$ and $R_{T}$ as a condition to be imposed on the viscosity according to

$$
\eta(T) \leq \eta^{*} \approx \mu \tau_{\mathrm{th}} \approx \mu R_{T}^{2} / D_{a} .
$$

The threshold viscosity $\eta^{*}$ may be considered to define an effective flow temperature $T^{*}$ as introduced in Refs. [15-18]. For values of $\mu$ of the order of $100 \mathrm{GPa}$ and values of $\tau_{\text {th }}$ between $10^{-11}$ and $10^{-9} \mathrm{~s}, \eta^{*}$ is estimated to range from 1 to $100 \mathrm{Pas}$. In well relaxed amorphous materials, this requires temperatures around the melting point.

For moderate values of $S_{e}$, such high temperatures (near the melting temperature) may not be reached in the track meaning that no local shear stress relaxation and consequently no macroscopic deformation would take place in such cases. But the viscosity and the corresponding threshold flow temperature may be reduced by an accumulation of "flow defects" resulting from atomic displacements during irradiation. It was assumed [14] that such a modification of the irradiated amorphous solid may open low activation-energy modes of the viscous flow which are locked in a relaxed amorphous alloy. This idea is supported by the following experimental observations: (1) For (unirradiated) samples of the amorphous metallic alloy $\mathrm{Pd}_{77.5} \mathrm{Cu}_{6} \mathrm{Si}_{16.5}$, the apparent activation energy varies from $2.0 \mathrm{eV}$ down to $0.4 \mathrm{eV}$ at $T \leq 535 \mathrm{~K}$ depending on the annealing conditions [26]. (2) The incubation dose for anisotropic growth of the amorphous metallic alloy $\mathrm{Pd}_{80} \mathrm{Si}_{20}$ is significantly reduced by cold-rolling and this reduction can be removed by annealing [5].

The joint effect of defect accumulation in the matrix and local material heating in the track results in a dramatic decrease of the viscosity down to its threshold value $\eta^{*}$. The threshold defect concentration required to reach $\eta^{*}$ defines the incubation fluence. Because the defects created during the passage of a heavy ion can annihilate after the track cooling, their accumulation does not take place at sufficiently high irradiation temperature $T_{\text {irr }}$ (ambient temperature). Following Tsao and Spaepen [26] we write the fluidity $\eta^{-1}$ resulting from flow defects of atomic concentration $n_{f}$ as

$$
\eta^{-1}=\left(\gamma^{2} \Omega_{f} n_{f} \nu / k_{B} T\right) \exp \left(-G / k_{B} T\right),
$$

where $\Omega_{f}$ is the volume of a flow region, i.e., the region consisting of a defect and its immediate surrounding; $G$ is the activation energy of local atomic rearrangements in the flow defect resulting in a local shear strain $\gamma$.

In the low fluence range (low dose) of linear defect accumulation, the flow-defect concentration can be expressed as

$$
n_{f}=\sigma_{f} \phi \varphi\left(T_{\mathrm{irr}}\right),
$$

where $\sigma_{f}=\sigma_{f}\left(S_{n}, S_{e}\right)$ is the flow-defect production cross section to which elastic collisions as well as electronic excitations contribute; $\varphi\left(T_{\text {irr }}\right)$ takes into account the defect relaxation after the track cools to the vicinity of the irradiation temperature $T_{\mathrm{irr}}$, with $\varphi\left(T_{\mathrm{irr}}\right) \rightarrow 1$ at low values of $T_{\text {irr }}$ and $\varphi\left(T_{\text {irr }}\right) \rightarrow 0$ when $T_{\text {irr }}$ is high. The corresponding viscosity decrease is given by Eq. (3). As soon as the viscosity in the heated track reaches the threshold value $\eta^{*}$, local stress relaxation and anisotropic growth will occur. Accordingly, the incubation fluence $\phi^{*}$ may be defined as

$$
\eta=\eta\left[n_{f}\left(\phi_{c}\right), T_{0}\right]=\eta^{*},
$$

where $T_{0}=T_{\text {irr }}+\Delta T_{0}$ is the maximum temperature in the center of the ion track according to Eq. (1).

Combining Eqs. (1), (3), (4), and (5), we obtain the following expression for the incubation fluence:

$$
\phi_{c}=\frac{\phi_{n}}{\sigma_{f}\left(S_{n}, S_{e}\right) \varphi}\left(T_{n}+S_{e}\right) \exp \left[G_{n} /\left(T_{n}+S_{e}\right)\right],
$$

where we have introduced normalized quantities $\quad T_{n}=3 \pi n_{a} k_{B} R_{T}^{2} T_{\mathrm{irr}}, \quad G_{n}=3 \pi n_{a} R_{T}^{2} G, \quad$ and 
$\phi_{n}^{-1}=3 \pi \gamma^{2} \nu \eta^{*} n_{a} \Omega R_{T}^{2}$, where $\Omega$ is the atomic volume. In Eq. (6), we have separated the functions depending strongly on $S_{e}$ from the function $\phi_{n}$ which depends only weakly upon $S_{e}$ via $\eta^{*}$.

For the estimated initial electron temperatures $\left(\varepsilon_{f}<T_{e}<5 \varepsilon_{f}\right.$, where $\varepsilon_{f}$ is the Fermi energy) [19-21] the electronic thermal diffusivity $D_{e}\left(T_{e}\right)$ has a deep flat minimum $\left[D_{e}\left(T_{e}\right) \approx 1-3 \mathrm{~cm}^{2} / \mathrm{s}\right]$. Moreover, in this range the characteristic time of the energy transfer due to electron-ion coupling $\tau_{e i}\left(T_{e}\right)$ also depends weakly on the electron temperature [23,24]. Therefore, in the electron temperature region $\epsilon_{f}<T_{e}<5 \epsilon_{f}$ the heating radius $R_{T}^{2} \simeq 4 D_{e} \tau_{e a}$ depends only weakly on the initial electron temperature and the electronic energy loss $S_{e}$ and we neglect below this dependence in Eq. (6). For amorphous $\mathrm{Fe}_{85} \mathrm{~B}_{15}$ we take $[19-21] T_{e}^{\max }=3 \epsilon_{F}, \epsilon_{F}^{(\mathrm{Fe})}=5 \mathrm{eV}, D_{e}=2.39 \mathrm{~cm}^{2} / \mathrm{s}$, $\tau_{e i}=1.34 \times 10^{-12} \mathrm{~s}$, and obtain $R_{T}=35 \mathrm{~nm}$ which is in a reasonable agreement for the heating radius $\left(R_{T}=20 \mathrm{~nm}\right)$ resulting from a fitting to the experimental data [6].

To our knowledge, the only system for which a satisfactory number of data are available for testing the stopping power dependence of the incubation dose is the amorphous metallic alloy $\mathrm{Fe}_{85} \mathrm{~B}_{15}$ [6] irradiated at low irradiation temperatures $\left[T_{\mathrm{irr}}=90 \mathrm{~K}, \varphi\left(T_{\mathrm{irr}}\right) \rightarrow 1\right]$. In these (as in other [5]) experimental studies, the "incubation dose" was determined by extrapolating the linear steady-state growth to vanishing total growth. This is justified for medium values of $S_{e}$ (5 to $30 \mathrm{keV} / \mathrm{nm}$ ) where the transition from the incubation behavior to linear growth is sharp.

Defect production has been studied in some detail in [7,8,27-29], in which the electronic excitations dominate

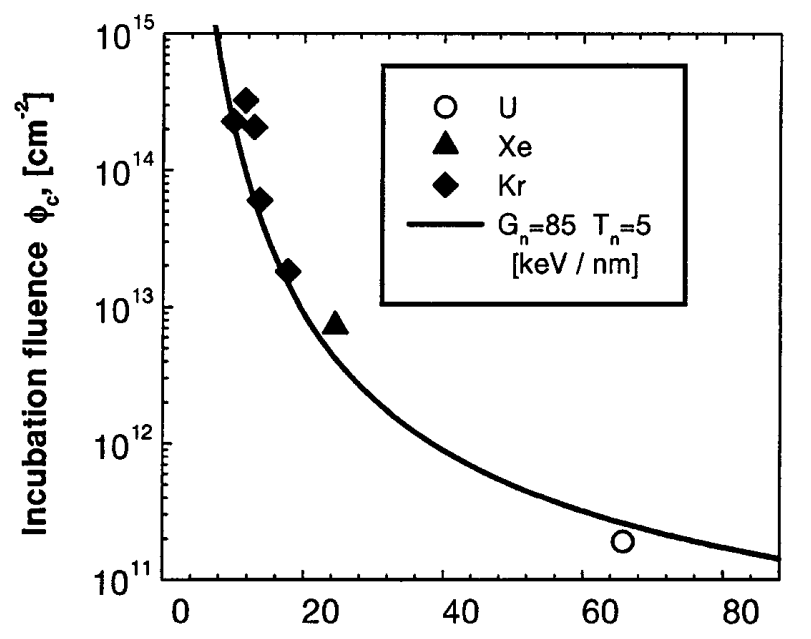

\section{Electronic stopping power $\mathbf{S}_{\mathbf{e}}$, $[\mathrm{keV} / \mathrm{nm}]$}

FIG. 1. Dependence of the incubation fluence $\left(\phi_{c}\right)$ on the electronic stopping power $\left(S_{e}\right)$. The curve shows a fit to experimental data [6] on the basis of Eq. (6). in the defect production cross section. We approximate these data here by $\sigma_{f}=5 \times 10^{-5} S_{e}^{2} \mathrm{~nm}^{4} / \mathrm{keV}^{2}$. Taking this into account we have fitted the experimentally observed $S_{e}$ dependence of $\phi_{c}$ according to Eq. (6). The result, shown in Fig. 1, yields the fit parameters $T_{n}=5 \mathrm{keV} / \mathrm{nm}, G_{n}=85 \mathrm{keV} / \mathrm{nm}$, and $\phi_{n}^{-1}=4 \times$ $10^{5} \mathrm{keV} / \mathrm{nm}$.

It has to be examined whether these values are reasonable. At $90 \mathrm{~K}, T_{n}$ yields a "track radius" of about $20 \mathrm{~nm}$ and a maximum temperature increase $\Delta T_{0}$ in the heated track of about $1150 \mathrm{~K}$ in reasonable agreement with a numerical study of such heating induced by electronic excitations in $\mathrm{Fe}_{85} \mathrm{~B}_{15}$ [21]. $G=G_{n} k_{B} T_{\text {irr }}$ yields about $0.3 \mathrm{eV}$ which lies in the range expected for such systems [26]. The large value of $G / k_{B} T=G_{n} / T_{n} \approx 20$ guaranties thermal stability of the created defects after the track cooling to $T_{\text {irr }}$. The value of $\phi_{n}$ is, for instance, consistent with $\gamma=2, \nu=10^{13} \mathrm{~s}^{-1}, \eta^{*}=1 \mathrm{Pas}$, and $R_{T}=20 \mathrm{~nm}$ which also appears reasonable. Thus, the model presented does not only describe the $S_{e}$ dependence of the incubation dose correctly but is also consistent with experimental observations and numerical calculations concerning the values of the parameters.

It is interesting here to consider the stopping power dependence of the product of the defect production cross section $\sigma_{f}$ and the incubation fluence $\phi_{c}$ (see Fig. 2). For the experimental values of this product, $\sigma_{f}$ is assumed to be proportional to the relative electrical resistivity change $(D)$ measured at the beginning of irradiation, $\sigma_{f} \propto D[6]$. Using the same values for $G_{n}$ and $T_{n}$ as above we find a

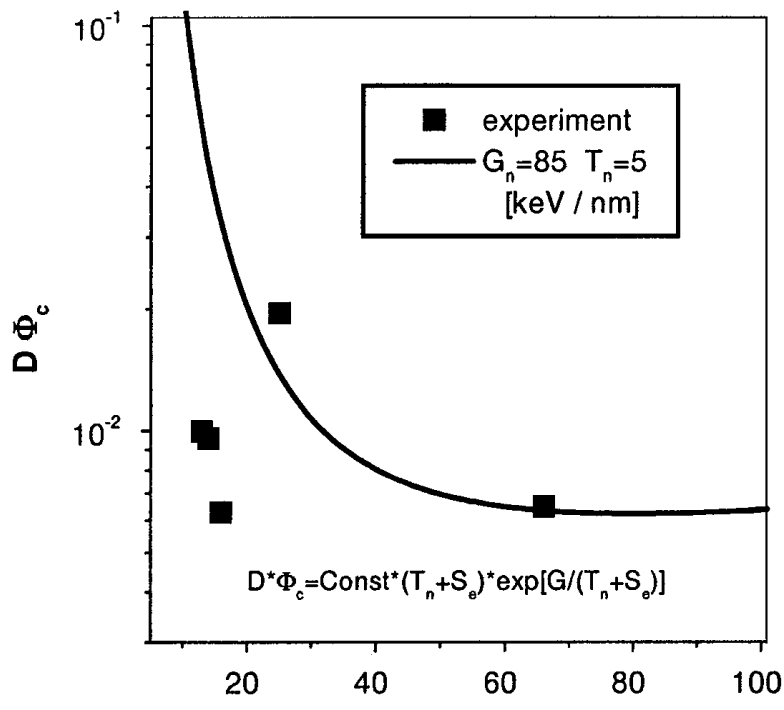

Electronic stopping power $\mathrm{S}_{\mathrm{e}},[\mathrm{keV} / \mathrm{nm}]$

FIG. 2. Dependence of the product of the incubation fluence $\left(\phi_{c}\right)$ and the relative electrical resistivity change at the beginning of the irradiation $\left(D \propto \sigma_{f} \varphi\right)$ on the electronic stopping power $\left(S_{e}\right)$. The curve shows a fit to experimental data [6] on the basis of Eq. (6). 
theoretical dependence of $D \phi_{c}$ which has a flat minimum at a relevant high $S_{e}$ of $80 \mathrm{keV} / \mathrm{nm}$. The predicted $S_{e}$ dependence of $D \phi_{c}$ becomes strong only at low $S_{e}$ where the experimental accuracy becomes low. Accordingly, the $S_{e}$ dependence of $\phi_{c}$ is mainly due to that of $\sigma_{f}$ in the range of experimental values shown in Fig. 2. On the other hand, Fig. 2 shows that more accurate measurements in the low $S_{e}$ are needed to test the details of the model.

The dependence of the incubation fluence on the irradiation temperature results from two effects acting in opposite directions. On the one hand, at high irradiation temperatures $\left(T_{\mathrm{irr}}>300 \mathrm{~K}\right)$ the flow defect accumulation in the matrix is suppressed by defect recombination after the track cooling $\left[\varphi\left(T_{\mathrm{irr}}\right) \rightarrow 0\right]$ resulting in an increase of the incubation fluence with increasing the irradiation temperatures. On the other hand the efficiency of the flow defects as described by the term $\left(T+S_{e}\right) \exp \left[G_{n} /\left(T+S_{e}\right)\right]$ increases with $T_{\text {irr }}$ (see Fig. 2) resulting in a decrease of $\phi_{c}$ with increasing $T_{\mathrm{irr}}$. Unfortunately, experimental measurements of the incubation fluence at only two irradiation temperatures are available [6] and both of them fall into the range where the incubation fluence decreases with increasing irradiation temperature.

In summary, we may conclude that the delayed onset of anisotropic growth and particularly its electronic stopping power dependence may be attributed to a reduction of the viscosity by the production of flow defects under irradiation. We think that more detailed and accurate measurements of the defect production, the stopping power, and the temperature dependence of the incubation dose and of the steady-state growth rate in combination with refined modeling should be able to provide deeper insight into amorphous-state modifications. Modified states could be tested at time scales and temperatures not accessible to conventional methods.

*Corresponding author: tel. +7 (095) 1969766

fax. +7 (095) 1969177

Email address: volkov@dni.polyn.kiae.su

[1] Metallic Glasses, edited by J. J. Gilman and H. J. Leamy (American Society for Metals, Metals Park, OH, 1978).

[2] S. Klaumünzer and G. Schumacher, Phys. Rev. Lett. 51, 1987 (1983).

[3] S. Klaumünzer, G. Schumacher, Ming-dong Hou, and G. Vogl, in Proceedings of the 5th International Confer- ence on Rapidly Quenched Metals, edited by S. Steeb and H. Warlimont (North-Holland, Amsterdam, 1985), p. 895.

[4] S. Klaumünzer, Ming-dong Hou, and G. Schumacher, Phys. Rev. Lett. 57, 850 (1986).

[5] Ming-dong Hou, S. Klaumünzer, and G. Schumacher, Nucl. Instrum Methods Phys. Res., Sect. B 19/20, 16 (1987).

[6] A. Audouard, E. Balanzat, J. C. Jousset, D. Lesueur, and L. Thome, J. Phys. Condens. Matter 5, 995 (1993).

[7] A. Audouard, J. Dural, M. Toulemonde, A. Lovas, G. Szenes, and L. Thome, Nucl. Instrum. Methods Phys. Res., Sect. B 107, 185 (1996).

[8] A. Audouard, M. Toulemonde, G. Szenes, and L. Thome, Nucl. Inst. Methods Phys. Res., Sect. B 146, 233 (1998).

[9] C. Hardtke, W. Schilling, and H. Ullmaier, Nucl. Instrum. Methods Phys. Res., Sect. B 59/60, 377 (1991).

[10] C. A. Volkert, J. Appl. Phys. 70, 352 (1991).

[11] A. Barbu, M. Bibole, R. Ie Hazit, S. Bouffard, and J. C. Ramilou, J. Nucl. Mater. 165, 217 (1989).

[12] E. Snoeks, A. Polman, and C. A. Volkert, Appl. Phys. Lett. 65, 2487 (1994).

[13] E. Snoeks, T. Weber, A. Caceiato, and A. Polman, J. Appl. Phys. 78, 4723 (1995).

[14] A. I. Ryazanov, A.E. Volkov, and S. Klaumünzer, Phys. Rev. B 51, 12107 (1995).

[15] H. Trinkaus and A. I. Ryazanov, Phys. Rev. Lett. 74, 5072 (1995).

[16] H. Trinkaus, Nucl. Instrum. Methods Phys. Res., Sect. B 107, 155 (1996).

[17] H. Trinkaus, Nucl. Instrum. Methods Phys. Res., Sect. B 146, 204 (1998).

[18] H. Trinkaus, J. Nucl. Mater. 223, 169 (1995).

[19] Yu. V. Martynenko and Yu. N. Yavlinskii, Sov. Phys. Dokl. 28, 391 (1983).

[20] M. Toulemonde, E. Paumier, and Ch. Dufour, Radiat. Eff. Defects Solids 126, 201 (1993).

[21] C. Dufour, CEA Report No. CEA-R-5638, 1993.

[22] G. Szenes, Nucl. Instrum. Methods Phys. Res., Sect. B 107, 150 (1996).

[23] A. E. Volkov and V. A. Borodin, Nucl. Instrum. Methods Phys. Res., Sect. B 107, 127 (1996).

[24] A. E. Volkov and V. A. Borodin, Nucl. Instrum. Methods Phys. Res., Sect. B 146, 137 (1998).

[25] M. I. Kaganov, I. M. Lifshitz, and L. V. Tanatarov, Sov. Phys. JETP 4, 173 (1957).

[26] S. S. Tsao and F. Spaepen, Acta Metall. 33, 891 (1985).

[27] A. Dunlop and D. Lesueur, Radiat. Eff. Defects Solids 126, 123 (1993).

[28] A. Audonard, E. Balanzat, G. Fuchs, J.C. Jousset, D. Lesueur, and L. Thome, Europhys. Lett. 3, 327 (1987).

[29] A. Audouard, E. Balanzat, G. Fuchs, J. C. Jousset, D. Lesueur, and L. Thome, Europhys. Lett. 5, 241 (1988). 